\title{
CURRENT SINGULARITIES AT QUASI-SEPARATRIX LAYERS AND THREE-DIMENSIONAL MAGNETIC NULLS
}

\author{
I. J. D. CRAig ANd Frederic EFFENBERGER \\ Department of Mathematics, University of Waikato, P.B. 3105, Hamilton, New Zealand; feffen@ waikato.ac.nz \\ Received 2014 July 16; accepted 2014 September 17; published 2014 October 22
}

\begin{abstract}
The open problem of how singular current structures form in line-tied, three-dimensional magnetic fields is addressed. A Lagrangian magneto-frictional relaxation method is employed to model the field evolution toward the final near-singular state. Our starting point is an exact force-free solution of the governing magnetohydrodynamic equations that is sufficiently general to allow for topological features like magnetic nulls to be inside or outside the computational domain, depending on a simple set of parameters. Quasi-separatrix layers (QSLs) are present in these structures and, together with the magnetic nulls, they significantly influence the accumulation of current. It is shown that perturbations affecting the lateral boundaries of the configuration lead not only to collapse around the magnetic null but also to significant QSL currents. Our results show that once a magnetic null is present, the developing currents are always attracted to that specific location and show a much stronger scaling with resolution than the currents that form along the QSL. In particular, the null-point scalings can be consistent with models of "fast" reconnection. The QSL currents also appear to be unbounded but give rise to weaker singularities, independent of the perturbation amplitude.
\end{abstract}

Key words: magnetic reconnection - magnetohydrodynamics (MHD) - methods: numerical - Sun: flares Sun: magnetic fields

Online-only material: color figures

\section{INTRODUCTION}

Magnetic reconnection is the mechanism that allows topological change in weakly resistive magnetic plasmas such as the solar corona. For reconnection to be effective, huge currents involving steep field gradients must be present. How these near-singular current structures develop is not fully understood, but it is generally recognized that topological features of the field - null points and separators-should play a decisive role (Lau \& Finn 1990; Priest \& Titov 1996; Pontin \& Craig 2006). More physically, these features are likely to provide sites for the quiescent heating of the corona and the rapid energy release in solar flares (Parker 1972).

One route to understanding reconnection is to examine the eigenstructure of three-dimensional (3D) magnetic nulls. This provides a field skeleton that comprises "spine" lines and "fan" planes that accumulate current when the null is suitably perturbed. For instance, bending the spine of an isolated X-point leads to a current layer aligned to the fan, i.e., "fan" reconnection (for a review on topological aspects in reconnection, see, e.g., Longcope 2005). However, a different current structure emerges when the fan plane is distorted. This leads to "spine" reconnection in which quasi-cylindrical tubes of current become localized to the spine axis (Craig \& Fabling 1996).

Current sheet formation and reconnection can also take place in the absence of a null. The key feature in this case is the "quasi-separatrix layer" (QSL; Priest \& Démoulin 1995; Titov et al. 2002; Démoulin 2006; Aulanier et al. 2006). This is a region of rapid variation in field-line connectivity that can be thought of as being of geometrical (Titov \& Hornig 2002) rather than topological significance. The simplest example is provided by a line-tied planar X-point threaded by uniform axial field. The QSL extends between the upper and lower planes $z= \pm L$ (say) and replaces the separatrix surfaces of the purely planar
X-point. All points on the QSL are connected Alfvénically, but there is no unique point, like a magnetic null, on which currents can accumulate (Galsgaard 2000; Craig \& Pontin 2014).

There is observational evidence that current formation and reconnection involving coronal active region outflows are connected to QSLs (Baker et al. 2009; Guo et al. 2013). Models of solar flares (Demoulin et al. 1996; Zhao et al. 2014) and coronal mass ejections also suggest that QSLs can play a decisive role in the initiation of such eruptions (Schrijver et al. 2011; Savcheva et al. 2012). More theoretically, since QSLs provide strong layers of currents, they must be regarded as prime sites for particle acceleration in the active corona (see Heerikhuisen et al. 2002; Stanier et al. 2012).

How QSLs influence reconnection is not entirely clear. Opinion is divided on whether disturbances that include shifts of the line-tied boundary can initiate a collapse to an ideal current singularity -as they do in null-point reconnection-or whether very steep but finite current distributions are obtained (Titov et al. 2003; Galsgaard et al. 2003). What is known, at least for magnetic X-points, is that by strengthening the effects of axial line-tying either by increasing the axial field or shortening the distance between the upper and lower linetied boundaries, current localization can be inhibited (Craig \& Pontin 2014). This leads to the strongest current accumulation around the outer boundaries of the X-point, which is unfavorable to rapid reconnection and artificial in terms of a computational reconnection experiment.

In order to prescribe an initial field in which QSL structures or magnetic nulls are present, a potential field generated by submerged magnetic monopoles is often considered. Studies of these magnetic structures, driven by slow lower-boundary photospheric motions, show strong currents developing along the QSL with no signs of saturation in the accessible numerical resolution regime (Aulanier et al. 2005; Effenberger et al. 
2011). The strong gradients due to the monopoles that are situated closely below the lower boundary can, however, present numerical difficulties that may artificially result in the largest currents forming close to that boundary.

The aim of the present study is to compare QSL current structures with those in isolated magnetic nulls. In contrast to previous studies based on the submerged monopole approach, we use line-tied, analytically prescribed fields that allow for a continuous transformation between null-point and QSL equilibria. This allows null-point and QSL currents to be treated on the same physical footing. In Section 2, we discuss the form of the general force-free equilibrium field we employ as the basis of our study and detail how perturbations can lead to current formation. In Section 3, we will present results from relaxation runs performed with both a linearized, potential version of this field and the more general force-free field. We finally discuss our findings in Section 4.

\section{GENERALIZED QSL AND X-POINT FIELDS}

We consider an equilibrium field $\mathbf{B}(\mathbf{r})$ defined within the interior of the rectangular domain $\mathcal{D}$ and line tied on all the bounding surfaces. This field is subject to some finite amplitude disturbance that displaces the footpoints of the equilibrium field altering the magnetic topology. Magnetic reconnection allows the perturbed field to "relax" dynamically into a new equilibrium, which is topologically different from the initial disturbed field. However, if resistive effects are absent but some other form of damping is present, the perturbed field has to relax without topological change. In this case, a near-singular configuration can emerge, comprising strong, highly localized current densities. It is the properties and definition of these nearsingular "relaxed" fields that are the focus of the present study.

Our starting point is an initial field $\mathbf{B}(\mathbf{r})$ with the components

$$
\begin{gathered}
B_{x}=\kappa \mu x \cos (\mu z-b)+(1-\kappa) \mu y \sin (\mu z-b), \\
B_{y}=(1-\kappa) \mu y \cos (\mu z-b)-\kappa \mu x \sin (\mu z-b), \\
B_{z}=-\sin (\mu z-b) .
\end{gathered}
$$

We assume that field intensities and distances are scaled according to typical coronal values, for example, $10^{2} \mathrm{G}$ for the magnetic field and $10^{9.5} \mathrm{~cm}$ for the coronal size scale. The domain $\mathcal{D}$ is then the region $-1, \leqslant x, y, z \leqslant 1$.

The initial field is defined by the three parameters, $\mu, \kappa$, and $b$, and has the following properties:

$$
\begin{aligned}
\nabla \cdot \mathbf{B} & =0, \\
\nabla \times \mathbf{B} & =\mu \mathbf{B}, \\
\nabla^{2} \mathbf{B} & =-\mu^{2} \mathbf{B} .
\end{aligned}
$$

We see that $\mu$ accounts for a rotation phase, while $\kappa \neq 1 / 2$ allows for rotational asymmetries about the $z$-axis in the field. The null is located at the point

$$
\mathbf{r}_{p}=\frac{b}{\mu} \hat{\mathbf{z}}
$$

and, for fixed $\mu$, can be shifted outside the field domain by adjusting $b$. The field, being force-free, is considerably more general than potential fields due to the presence of parallel currents.

\subsection{Related Potential Fields}

Potential fields can be extracted from the general field (Equation (3)) by formally regarding $\mu$ and $b$ as sufficiently small parameters. We then obtain the linear potential field

$$
\mathbf{P}_{1}=(\kappa \mu x,(1-\kappa) \mu y, b-\mu z) .
$$

We can avoid redundant parameterization by taking $\kappa=1 / \mu$ :

$$
\mathbf{P}_{2}=(x,(\mu-1) y,-\mu z+b) .
$$

In this case, we should regard $\mu$ as a proxy for the field asymmetry. Note that although the potential forms allow considerable simplification, they can be expected to provide a reasonable guide to the current accumulation properties of the more general field (Equation (3)), at least in regions close to the null. This point is revisited in Section 3 below.

\subsection{Spines, Fans, and QSLs}

In our analysis, we consider only perturbing fields $\mathbf{B}_{p}$ that disturb the lateral boundaries of the domain. To illustrate the effect of these disturbances, consider the simple form

$$
\mathbf{B}_{p}=\left(0, a_{1} x, a_{2} x\right)
$$

in the superposition

$$
\begin{aligned}
\mathbf{B}_{s} & =\mathbf{P}_{2}+\mathbf{B}_{p} \\
& =\left(x,(\mu-1) y+a_{1} x, b-\mu z+a_{2} x\right) .
\end{aligned}
$$

Provided that $a_{1}$ or $a_{2}$ is non-zero, the perturbation is finite on the boundary points $x= \pm 1$. The field-line equations $\mathbf{d r} \propto \mathbf{B}_{s}$ in the case of a null-point field give (assuming $\mu \neq 0$ )

$$
x^{1-\mu}\left(y-\frac{a_{1} x}{2-\mu}\right)=C_{1}, \quad x^{\mu}\left(z-\frac{b}{\mu}-\frac{a_{2} x}{\mu+1}\right)=C_{2},
$$

where $C_{1}$ and $C_{2}$ are constants. The separatrices are defined by field lines that thread the null, i.e., by setting $C_{1}=C_{2}=0$. We obtain the "fan" plane $x=0$ and the "spine" line

$$
y=\frac{a_{1} x}{2-\mu}, \quad z=\frac{b}{\mu}+\frac{a_{2} x}{\mu+1} .
$$

We see that the spine line of the unperturbed field-the $x$-axis in the case $b=0-$ becomes tilted due to the perturbation. This generates currents of magnitude $\sqrt{a_{1}^{2}+a_{2}^{2}}$ within the fan. When no resistivity is present, these initial currents localize-and eventually blow up-in the vicinity of the null point.

The fan-spine structure breaks down when the null point is absent. In the simplest case of a planar field with no axial component ( $\partial_{z}=\mu=b=0$ ), the separatrices (obtained by the first of Equation (12)) are just the two planes $x=0$ and $y=0$, the latter being tilted through the angle $\tan \theta=a_{1} /(2-\mu)$ (see, e.g., Craig \& Pontin 2014). The null point is now extended to a null line defined by the intersection of the two planes.

In the case of a constant finite axial field ( $b \neq 0, \mu=0)$, the null line is removed and separatrices cannot be defined. Even so, each z-plane still retains a projected copy of the tilted separatrix planes of the perturbed planar X-point. Reconnection again requires currents localized toward the $z$-axis but now involves field lines whose ends are anchored across different 
$z$-planes. Line-tying the axial field on $z= \pm 1$, however, breaks the symmetry $\partial_{z}=0$ and makes the geometry fully $3 \mathrm{D}$. This is the topology of the QSL: no null is present but magnetic stresses can accumulate due to steep field gradients centered on the $z$-axis.

Finally, we turn to the field-line equations in the case $\mu=0$, that is, the simplest case of a QSL field (i.e., $B_{z}=b$ ), without a null:

$$
x\left(y-\frac{a_{1} x}{2}\right)=C_{3}, \quad x \exp \left(\frac{a_{2} x-z}{b}\right)=C_{4} .
$$

We see that there is strong exponential dependency on the $C_{4}$ field line even when the perturbation amplitude vanishes $\left(a_{2}=0\right)$. This dependency reflects the geometrical squashing factors associated with equilibrium field lines when no null is present (Titov 2007).

\subsection{Squashing Factors for QSLs}

As a complement to the computational study of Section 3, it is instructive to determine QSL squashing factors for the case where $a_{2}$ vanishes but $a_{1}$ remains finite. In this case, we regard the upper and lower boundaries as adjustable planes $z= \pm z_{m}$ (say) and use the field-line equations (Equations (14)) to relate the upper and lower footpoint locations. Denoting the footpoint coordinates at the upper and lower boundaries with $x_{ \pm}$and $y_{ \pm}$, we have that

$$
\left(\begin{array}{c}
x_{+} \\
y_{+}
\end{array}\right)=\left(\begin{array}{cc}
\exp \left(2 \frac{z_{m}}{b}\right) & 0 \\
a_{1} \sinh \left(2 \frac{z_{m}}{b}\right) & \exp \left(-2 \frac{z_{m}}{b}\right)
\end{array}\right)\left(\begin{array}{c}
x_{-} \\
y_{-}
\end{array}\right) .
$$

These expressions can be used to determine the invariant squashing factor $Q$ along the tube axis as defined in (Titov 2007). We find that

$$
Q=2 \cosh \left(4 \frac{z_{m}}{b}\right)+a_{1}^{2} \sinh ^{2}\left(2 \frac{z_{m}}{b}\right) .
$$

For modest perturbation amplitudes (i.e., $\left|a_{1}\right|<1$ ), the squashing factor is determined mainly by the form of the equilibrium field; specifically, $Q$ increases rapidly with the tube length $\left(2 z_{m}\right)$ but decreases with the strength of the axial field. Given these properties, it seems natural to suppose that current accumulation in the QSL might reflect this behavior. There is some numerical evidence that longer tubes can lead to stronger current localizations along the tube axis (Craig \& Pontin 2014) but, to our knowledge, a direct link between QSL currents and squashing factors has not yet been established.

\section{COMPUTATIONAL RESULTS CURRENT FORMATION}

The previous discussion suggests that reconnection can be expected within QSL configurations in response to footpoint displacements of the lateral boundaries, like those given by Equation (10). What is less certain is the strength and location of the reconnection currents within the QSL. One possibility is that in addition to the form and amplitude of the footpoint displacements, current strengths may be moderated by geometric squashing factors (Titov 2007) associated with the equilibrium configuration. More specifically, given that the present computational setup reflects the disturbed field $\mathbf{B}_{s}$ of Section 2.2, we expect to see steep field gradients aligned to the $z$-axis of the domain.

To investigate further, we now compare the current structures of perturbed magnetic field equilibria, both in the presence and

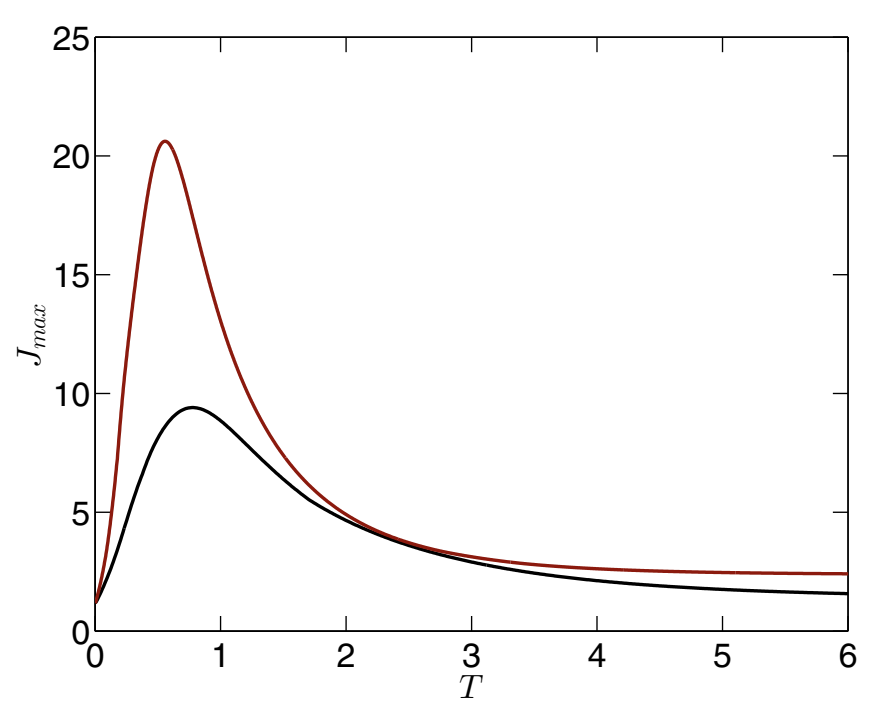

Figure 1. Pseudo-time evolution of the maximum current density during the relaxation to a force-free equilibrium for two different resolutions ( $N=61$, black and $N=81$, red). Time $T$ has been independently normalized in both cases to give comparable values of order one.

(A color version of this figure is available in the online journal.)

in the absence of a null point. We use the magneto-frictional Lagrangian scheme of Craig \& Pontin (2014), which models line tying by fixing fluid elements on the boundary of the domain $\mathcal{D}$. In practice, we adopt a uniform distribution of $N^{3}$ fluid particles in the interior of $\mathcal{D}$ and follow their evolution according to the Lorentz forces on the plasma. The code is implicit and unconditionally stable. The solenoidal condition $\nabla \cdot \mathbf{B}=0$ is satisfied to machine accuracy and flux and magnetic helicity are conserved. Gas pressure forces are neglected in the runs that follow.

The perturbation field we adopt is based on the simple model (see Equation (10)):

$$
\begin{aligned}
\mathbf{B}_{p}= & {\left[A \sin (\pi x / 2) \cdot\left(1-y^{2}\right) \cdot\left(1-z^{2}\right)\right.} \\
& \left.\cdot \exp \left(-4 x^{2}-3 y^{2}\right)\right] \hat{\mathbf{y}}
\end{aligned}
$$

where $A$ denotes the perturbation amplitude. The perturbation vanishes on all boundary points except $x= \pm 1$. Note that because the frictional relaxation can lead to divergent current structures, the computed values of certain local variables may be sensitive to numerical resolution. This makes it possible to obtain scaling laws in which the maximum current density in the domain $\mathcal{D}$ can be systematically quantified as a function of the resolution, i.e., the linear number $N$ of fluid particles in the domain.

\subsection{Relaxation of the Current Density}

The relaxation of the maximum current density in the domain for typical runs based on field $\mathbf{P}_{2}$ with $b=0.3$ and $\mu=-0.4$ are displayed in Figure 1 for two numerical resolutions, namely $N=61$ and $N=81$. The pseudo-time parameter $T$ is based on uniform time increments and reflects the number of iterations in the relaxation. In practice, the perturbation amplitude is chosen so that the initial forces and currents are of order of unity ( $A=0.3$ in the present runs) and the computation is halted when forces are reduced by four orders of magnitude. This protocol allows well-defined scaling laws of the form $J_{\max }=a_{0} N^{\alpha}$, where $a_{0}$ and $\alpha$ are constants and $J_{\max }$ is the 


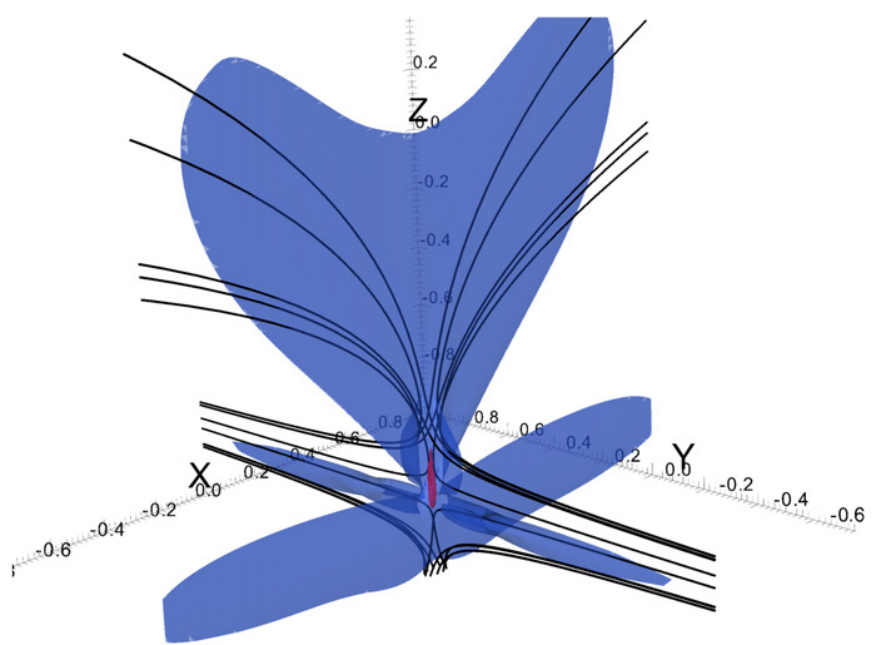

Figure 2. Current density distribution and field lines in the relaxed state for $b=0.3$ and $N=81$. The blue contour gives an isosurface of $J=0.1$ and the red contour is for $J=1$. The field lines show the QSL structure and the topology around the null at $z=-0.75$ where the strongest currents accumulate.

(A color version of this figure is available in the online journal.)

final maximum current density in the domain. Detailed scaling laws are discussed in Section 3.3 below.

Returning to Figure 1, it is clear that higher-resolution runs are associated with higher relaxed current densities. Furthermore, an even higher transitory current peak is visible in both cases. We do not investigate this phenomenon further in this study since we are interested mainly in the near-singular, relaxed state. It seems worth remarking, however, that resistive relaxation in a full magnetohydrodynamic (MHD) configuration often involves inertial overshoots that lead to oscillatory null-point reconnection (see, e.g., Craig \& McClymont 1991; Craig \& Watson 1992). It is likely therefore that the large transient currents in the pseudo-time evolution may reflect the strong initial implosion of the disturbance field toward the null.

\subsection{QSL versus Null-point Structure}

In the runs of Figure 1, the null point lies inside the computational domain $\mathcal{D}$ at the point $(0,0,-3 / 4)$, following from our parameter choice $b=0.3, \mu=-0.4$. We expect therefore to see current density distributions concentrated about the null. Figure 2 confirms this expectation, as illustrated by the red isosurface. The black field lines give the fan structure around the null, aligned roughly to the $y-z$ plane. The blue isosurface legs of weaker current, aligned to the $x$ - and $y$-axes, respectively, indicate that spine and fan currents form simultaneously (see the exposition in Section 2.2). Further away from the null, in the upper part of the domain, the field lines illustrate the additional QSL structure of the field associated with steep fieldline gradients aligned to the $z$-axis. The blue isosurface confirms the simultaneous formation of currents along the QSL with a magnitude similar to the fan and spine currents.

In Figure 3, we have changed the axial field parameter to $b=0.5$ so that the null now lies outside of the computational box. The QSL structure is still well represented and a salient feature is the "exponential" squashing the field lines toward the base, as suggested by the $C_{4}$ field lines of Equation (14). The currents are now considerably weaker than the peak current at the null of the previous setup. Since there is no preferred location along the QSL, the currents are more evenly distributed, which can be expected, however, not only from the absence of a null

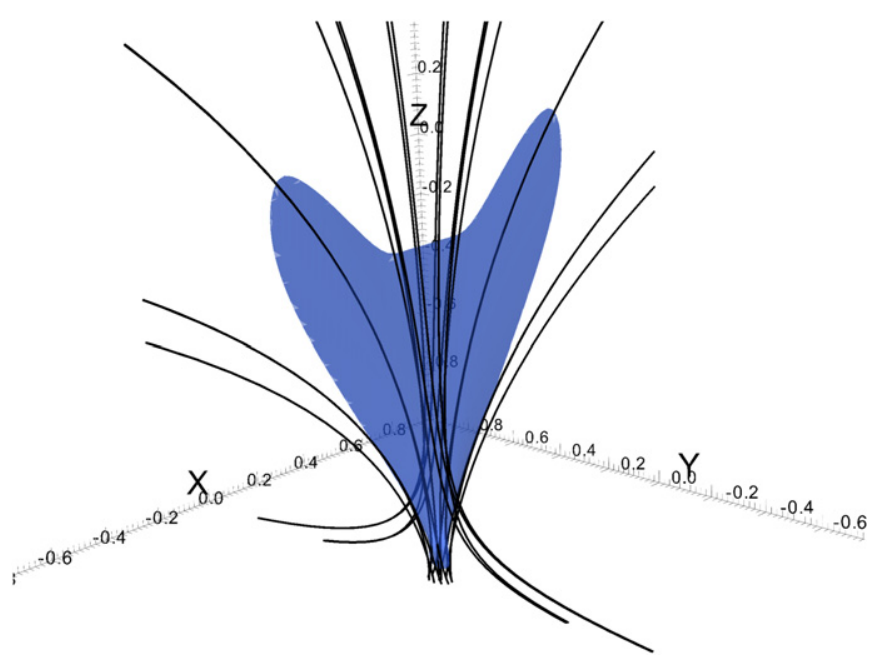

Figure 3. Similar to Figure 2 but now with $b=0.5$, i.e., no null present in the domain. The QSL structure is still visible but the current is weaker and more broadly distributed only along the QSL.

(A color version of this figure is available in the online journal.)

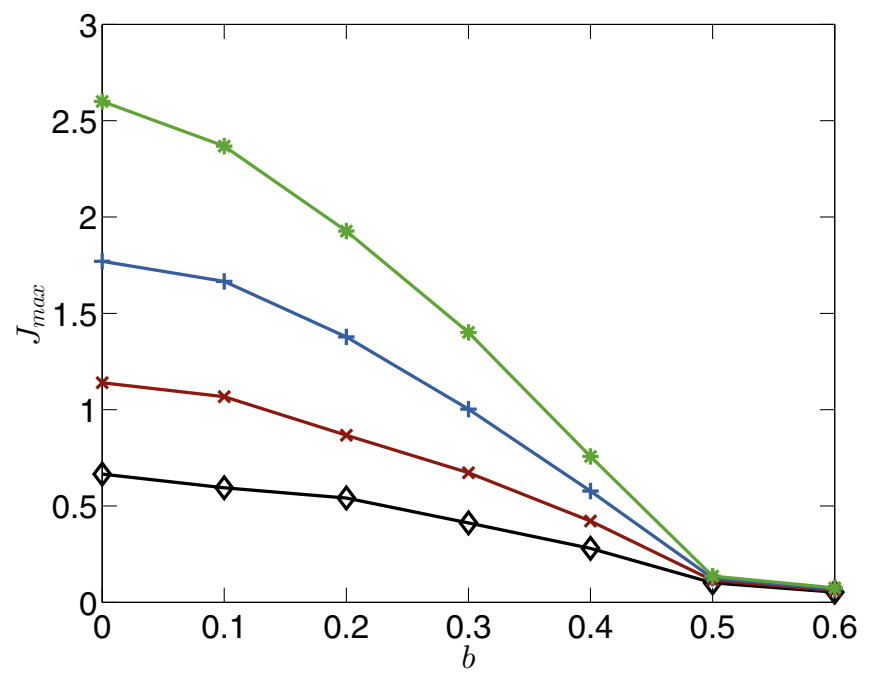

Figure 4. Current density $J_{\max }$ against $b$ for four different resolutions: $N=31$ (black, diamond), $N=41$ (red, x), $N=51$ (blue, + ), and $N=61$ (green, $*$ ). (A color version of this figure is available in the online journal.)

but also from the increased strength of the line-tied axial field that tends to resist current localization.

\subsection{Scaling of the Current Density}

To assess more systematically the influence of the axial field parameter $b$ on the maximum relaxed current density, we performed a series of runs with varying $b$ and resolution $N$ (keeping $\mu=-0.4$ in all cases). Figure 4 shows the dependence of $J_{\max }$ on the position of the null. The strongest currents develop for the weakest axial field and simultaneously largest distance of the null from the line-tied boundary. Once the null is outside the domain $(b=0.5)$, there is a visible change in the qualitative behavior of the current formation, which is a direct result of the field structure as already discussed in the previous section. The resolution dependence of the current is much weaker for these cases as well.

The quantitative scaling with resolution $N$ for different values of $b$ and thus null-point positions is given in Figure 5. This illustrates the variation of the relaxed maximum current density 


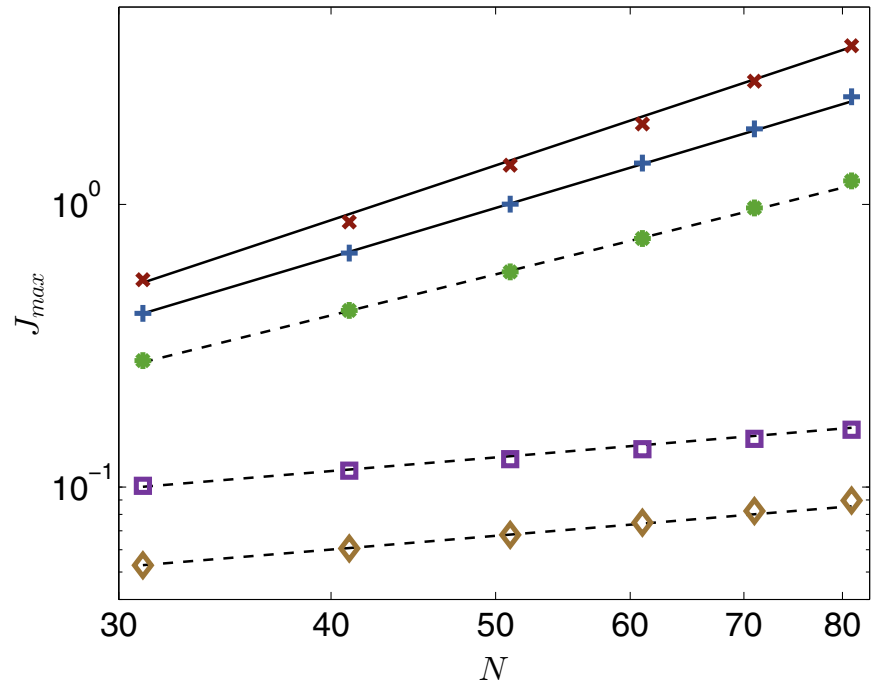

Figure 5. Current density $J_{\max }$ against $N$ for $b=0.2$ (red, x), $b=0.3$ (blue, + ), $b=0.4$ (green, $*$ ), $b=0.5$ (purple, box), and $b=0.6$ (brown, diamond). The solid lines indicate fits with strong scalings of $J_{\max } \propto N^{2.0}$ and $J_{\max } \propto N^{1.8}$, when the null is in the domain $(b=0.2$ and $b=0.3)$ and $J_{\max } \propto N^{1.5}$ where the null is right at the boundary $(b=0.4)$. The dashed lines give a weaker scaling fit of $J_{\max } \propto N^{0.5}$ for the two cases where the null is outside of the domain $(b=0.5$ and $b=0.6)$.

(A color version of this figure is available in the online journal.)

for a sequence of axial field strengths, specifically $b=0.2-0.6$ in increments of 0.1 . Since $\mu=-0.4$, the null is buried below the lower surface of $\mathcal{D}$ for the two runs where $b \geqslant 0.5$. We see a strong scaling with resolution close to $J_{\max } \propto N^{2}$ for the cases where the null is actually in the domain, with $b=0.4$ being the marginal case. For the QSL-only current formation, we only find a weak but still significant scaling of $J_{\max } \propto N^{1 / 2}$.

\subsection{Relation to Reconnection Models}

The question of what scaling should be expected for the peak current density with resolution is a key issue given that "fast" reconnection, i.e., reconnection independent of the weak coronal plasma resistivity, is thought to be required in solar flares. In fact, the $J_{\max }$ versus $N$ scalings of the previous section cannot provide reconnection rates by themselves. They can, however, be interpreted in light of fast reconnection models. In this case, the strong $J_{\max } \propto N^{2}$ scaling can be shown to be consistent with reconnection models in which the current sheet thickness scales linearly with the plasma resistivity (Petschek 1964; McClymont \& Craig 1996).

This is supported also by analytical reasoning in the simple case of a collapsing one-dimensional current sheet (with no axial field) modeled using a Lagrangian description (Craig \& Litvinenko 2005). We find in our numerical experiments that even for the fully $3 \mathrm{D}$ fields under consideration here, the limiting scalings $J_{\max } \propto N^{2}$ are reasonably approximated for cases where the null is well situated within the domain and are thus compatible with fast reconnection models. Conversely, it is hard to reconcile weaker scalings $J_{\max } \propto N^{0.5}$ with any known models of fast reconnection. It will be interesting to see, if the weak scaling results persist for, e.g., QSL fields constructed from submerged monopoles.

\subsection{The Force-free Field}

We now consider the full force-free field configuration given by Equation (3). Figure 6 gives the resulting relaxed current

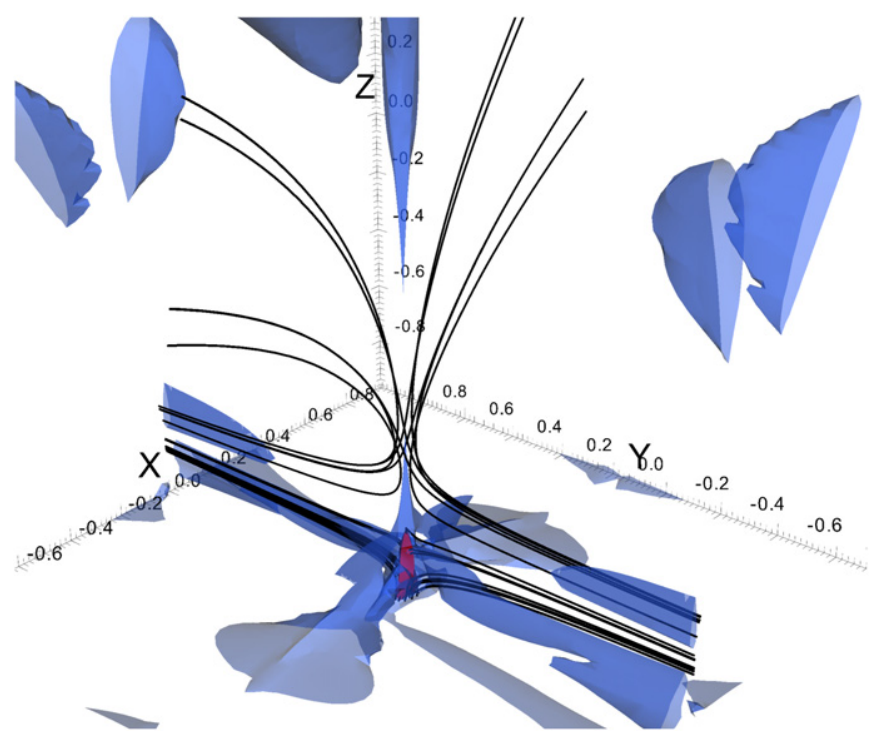

Figure 6. Current density distribution and field lines in the relaxed state for the initial force-free field (Equation (3)) configuration with $\kappa=-2.5, \mu=-0.4$, $b=0.3$, and $N=61$. The blue contour gives an isosurface of $J=0.8$ and the red contour is for $J=5$. The field lines show again the QSL structure and the topology around the null at $z=-0.75$.

(A color version of this figure is available in the online journal.)

configuration and magnetic field structure for a computational run with $\kappa=-2.5, \mu=-0.4, b=0.3$, and $N=61$. These values were chosen to give a close as possible configuration to the linear field studies presented above. As can be seen in the figure, the current again accumulates at the null, which is present at the same position as before, i.e., $(0,0,-3 / 4)$. The field structure around the null is also very similar, as should be expected from the linearization. The QSL currents, however, seem to be suppressed and overshadowed by the initial currents of the unperturbed field. Despite these differences, the field structure still shows the strong QSL field-line connectivity gradient. Exploratory computations with different resolutions indicate that the current magnitude at the null grows, as previously found in the linear field case, and reproduces a scaling $J_{\max } \propto N^{2}$ that is again consistent with fast reconnection. The analysis of current structure and formation is complicated, however, due to the currents already present in the initial forcefree configuration. We can see nonetheless that our linearized potential field model derived from the more complex force-free field can describe the structures and current build up close to the null with high accuracy.

\section{CONCLUSIONS}

We have investigated current formation in bounded line-tied magnetic field configurations using an ideal magneto-frictional relaxation method. Our initial fields include potential fields that derive from a general force-free field configuration. This approach allows us to distinguish a priori between fields that contain a magnetic null in the computational domain $\mathcal{D}$ and those that comprise only a strong QSL structure.

For parameter sets that comprise a magnetic null, we find that although the strongest currents are always attracted to the null, significant current layers can still form along the superimposed QSL structure. The QSL currents, however, are more strongly pronounced in the simpler, linear potential field than in the considerably more complicated structures of our general force-free field. The current formation along the QSLs 
becomes increasingly prominent for runs in which the null is positioned below the lower surface of the computational domain. For our range of parameters, the currents appear to spread evenly over the QSL. We believe this absence of focusing is due to a lack of an additional structure in the QSL at least in the fields we examine. This contrasts to, e.g., the hyperbolic flux tubes in some of the submerged monopole models (Aulanier et al. 2006; Effenberger et al. 2011). It would be of some interest therefore to extend our investigation to similar monopole field configurations. This requires a careful study of the initial conditions in the relaxation since the strong gradients at the boundary overlying the monopoles can prevent reconnective currents from localizing convincingly in the interior of the domain.

One advantage of the present relaxation scheme is that we can follow current formation in a strictly "ideal" (i.e., resistivityfree) fashion. This allows one to compute scaling laws for the current divergence against resolution. In particular, we have seen that when the null is centered in the computational domain, scalings can be derived $J_{\max } \propto N^{2}$ that are consistent with fast reconnection. The downside of this approach is that having no access to the actual dynamic evolution toward the relaxed state, we cannot draw any definite conclusions on the time dependence of the current build up. Thus, dynamic effects like alignment between the velocity and magnetic field (Grauer \& Marliani 2000) that may come into play in the full MHD problem are not represented. We intend to investigate these and other dynamic effects further in the future by comparing results from the relaxation method as employed in this study with 3D ideal MHD calculations.

We thank the referee for useful comments that helped to improve the manuscript. This work was partially supported by the Marsden Fund of New Zealand.

\section{REFERENCES}

Aulanier, G., Pariat, E., \& Démoulin, P. 2005, A\&A, 444, 961

Aulanier, G., Pariat, E., Démoulin, P., \& DeVore, C. R. 2006, SoPh, 238, 347

Baker, D., van Driel-Gesztelyi, L., Mandrini, C. H., Démoulin, P., \& Murray, M. J. 2009, ApJ, 705, 926

Craig, I. J. D., \& Fabling, R. B. 1996, ApJ, 462, 969

Craig, I. J. D., \& Litvinenko, Y. E. 2005, PhPl, 12, 032301

Craig, I. J. D., \& McClymont, A. N. 1991, ApJL, 371, L41

Craig, I. J. D., \& Pontin, D. I. 2014, ApJ, 788, 177

Craig, I. J. D., \& Watson, P. G. 1992, ApJ, 393, 385

Démoulin, P. 2006, AdSpR, 37, 1269

Demoulin, P., Henoux, J. C., Priest, E. R., \& Mandrini, C. H. 1996, A\&A, 308, 643

Effenberger, F., Thust, K., Arnold, L., Grauer, R., \& Dreher, J. 2011, PhPl, 18,032902

Galsgaard, K. 2000, JGR, 105, 5119

Galsgaard, K., Titov, V. S., \& Neukirch, T. 2003, ApJ, 595, 506

Grauer, R., \& Marliani, C. 2000, PhRvL, 84, 4850

Guo, Y., Démoulin, P., Schmieder, B., et al. 2013, A\&A, 555, A19

Heerikhuisen, J., Litvinenko, Y. E., \& Craig, I. J. D. 2002, ApJ, 566,512

Lau, Y.-T., \& Finn, J. M. 1990, ApJ, 350, 672

Longcope, D. W. 2005, LRSP, 2,7

McClymont, A. N., \& Craig, I. J. D. 1996, ApJ, 466, 487

Parker, E. N. 1972, ApJ, 174, 499

Petschek, H. E. 1964, NASSP, 50, 425

Pontin, D. I., \& Craig, I. J. D. 2006, ApJ, 642, 568

Priest, E. R., \& Démoulin, P. 1995, JGR, 100, 23443

Priest, E. R., \& Titov, V. S. 1996, RSPTA, 354, 2951

Savcheva, A., Pariat, E., van Ballegooijen, A., Aulanier, G., \& DeLuca, E. 2012, ApJ, 750, 15

Schrijver, C. J., Aulanier, G., Title, A. M., Pariat, E., \& Delannée, C. 2011, ApJ, 738,167

Stanier, A., Browning, P., \& Dalla, S. 2012, A\&A, 542, A47

Titov, V. S. 2007, ApJ, 660, 863

Titov, V. S., Galsgaard, K., \& Neukirch, T. 2003, ApJ, 582, 1172

Titov, V. S., \& Hornig, G. 2002, AdSpR, 29, 1087

Titov, V. S., Hornig, G., \& Démoulin, P. 2002, JGRA, 107, 1164

Zhao, J., Li, H., Pariat, E., et al. 2014, ApJ, 787, 88 